\title{
Evaluation of tissue PCA3 expression in prostate cancer by RNA in situ hybridization- a correlative study with urine PCA3 and TMPRSS2-ERG
}

Joshua I Warrick ${ }^{1}$, Scott A Tomlins ${ }^{1,2,3,4}$, Shannon L Carskadon ${ }^{1,2}$, Allison M Young ${ }^{1}$, Javed Siddiqui ${ }^{1,2}$, John T Wei ${ }^{4}$, Arul M Chinnaiyan ${ }^{1,2,3,4,5}$, Lakshmi P Kunju ${ }^{1,2,3,6}$ and Nallasivam Palanisamy ${ }^{1,2,3,6}$

${ }^{1}$ Department of Pathology, University of Michigan Medical School, Ann Arbor, MI, USA; ${ }^{2}$ Michigan Center for Translational Pathology, University of Michigan Medical School, Ann Arbor, MI, USA; ${ }^{3}$ Comprehensive Cancer Center, University of Michigan Medical School, Ann Arbor, MI, USA; ${ }^{4}$ Department of Urology, University of Michigan Medical School, Ann Arbor, MI, USA and ${ }^{5}$ Howard Hughes Medical Institute, University of Michigan Medical School, Ann Arbor, MI, USA

\begin{abstract}
PCA3 is a prostate-specific non-coding RNA, with utility as a urine-based early detection biomarker. Here, we report the evaluation of tissue PCA3 expression by RNA in situ hybridization in a cohort of 41 mapped prostatectomy specimens. We compared tissue PCA3 expression with tissue level ERG expression and matched pre-prostatectomy urine PCA3 and TMPRSS2-ERG levels. Across 136 slides containing 138 foci of prostate cancer, PCA3 was expressed in $55 \%$ of cancer foci and $71 \%$ of high-grade prostatic intraepithelial neoplasia foci. Overall, the specificity of tissue PCA3 was $>90 \%$ for prostate cancer and high-grade prostatic intraepithelial neoplasia combined. Tissue PCA3 cancer expression was not significantly associated with urine PCA3 expression. PCA3 and ERG positivity in cancer foci was positively associated $(P<0.01)$. We report the first comprehensive assessment of PCA3 expression in prostatectomy specimens, and find limited correlation between tissue $P C A 3$ and matched urine in prostate cancer.

Modern Pathology (2014) 27, 609-620; doi:10.1038/modpathol.2013.169; published online 27 September 2013
\end{abstract}

Keywords: PCA3; prostate cancer; RNA in situ hybridization

PCA3 is a non-coding RNA specifically overexpressed in $>90 \%$ of prostate cancers. ${ }^{1,2}$ The urine assay for PCA3 (PROGENSA, Gen-Probe, San Diego, CA, USA) has recently been approved by the Food and Drug Administration for predicting prostate cancer on rebiopsy. As PCA3 is a non-coding RNA, immunohistochemical-based detection is not feasible. Only a single study evaluating tissue PCA3 RNA expression by in situ hybridization has been reported, which showed PCA3 expression in the

Correspondence: Dr LP Kunju, MD, Department of Pathology, 2G332 UH, 1500 East Medical Center Drive, Ann Arbor, MI 48104-5054, USA or Dr N Palanisamy, MSc, MPhil, PhD, Michigan Center for Translational Pathology, 2900 Huron Parkway, Traverwood IV, Suite 100, Ann Arbor, MI 48105, USA.

E-mail: lkunju@med.umich.edu or nallasiv@med.umich.edu ${ }^{6}$ These senior authors have contributed equally to this work. Received 15 May 2013; revised 19 July 2013; accepted 8 August 2013; published online 27 September 2013 majority of prostate cancer foci and high-grade prostatic intraepithelial neoplasia foci, as well as a substantial subset $(\sim 30 \%)$ of benign glands. ${ }^{3}$

In 2005, our group discovered chromosomal rearrangements in prostate cancer resulting in the fusion of the $5^{\prime}$ untranslated region of the androgen-regulated gene TMPRSS2 with members of the E26 transformation-specific family of transcription factors. ${ }^{4,5}$ Fusions involving ERG represent $90 \%$ of all E26 transformation-specific fusions, and fluorescence in situ hybridization for the detection of ERG rearrangements has been shown to be highly specific for prostate cancer and high-grade prostatic intraepithelial neoplasia adjacent to prostate cancer. ${ }^{6-9}$ Monoclonal antibodies against ERG have been used as a surrogate for ERG rearrangement status, and have demonstrated diagnostic utility with high sensitivity and specificity for prostate cancer with TMPRSS2-ERG rearrangements. ${ }^{10,11}$ 
Our group recently evaluated a clinical grade transcription-mediated amplification assay that quantifies TMPRSS2-ERG mRNA in post-digital rectal examination urine. We have shown that urine TMPRSS22-ERG in combination with urine PCA3 increases the utility of serum PSA to predict the presence of prostate cancer and significant prostate cancer (per Epstein criteria) upon rebiopsy and correlates with total linear dimension of $E R G$-positive prostate cancer foci on prostatectomy specimens. ${ }^{12}$

Unlike $E R G$, tissue level expression of PCA3 and its correlation with urine expression are not well understood. Therefore, to assess the tissue level expression pattern of $P C A 3$, we used a novel RNA in situ hybridization assay for the detection of $P C A 3$ and evaluated its expression in prostate cancer and high-grade prostatic intraepithelial neoplasia. We also compared tissue PCA3 expression with tissue ERG expression by immunohistochemistry, and urine expression of PCA3 and TMPRSS2-ERG.

\section{Materials and methods}

\section{Study Cohort}

The Institutional Review Board approved prostatectomy cohort studied (41 men who underwent prostatectomy at our institution between 2008 and 2011) was identified from a previously reported cohort of 301 men referred for prostate needle biopsy at the University of Michigan Health System. All cases were assessed for urine TMPRSS2-ERG and urine $P C A 3$ scores by transcription-mediated amplification as previously described. ${ }^{12,13}$ None of these patients received preoperative radiation or androgen deprivation therapy.

\section{Urine TMPRSS2-ERG and PCA3 Assays}

Urine TMPRSS2-ERG and PCA3 scores were determined as described previously. ${ }^{12,13}$ In short, urine specimens were obtained immediately after attentive digital rectal examination. Expression levels of TMPRSS2-ERG mRNA and PSA mRNA were determined by a third generation, final clinical transcription-mediated amplification assay. ${ }^{12-15}$ The PROGENSA PCA3 assay (Gen-Probe) was used to determine the PCA3 RNA and PSA mRNA urine levels. $P C A 3$ score was determined as a ratio of $P C A 3$ RNA to PSA mRNA. ${ }^{16}$

\section{Prostatectomy Evaluation}

Fresh prostates removed after surgery were weighed, measured, inked, and fixed in 10\% neutral formalin. Seminal vesicles, apex, and base were amputated and the remaining prostate was serially sectioned at $4-5 \mathrm{~mm}$ intervals perpendicular to the long axis of the gland from the base to apex and quartered. All prostatectomy specimens were reviewed by the study pathologists LPK and JIW. Tumor maps were generated by tracking each section and reconstructing them as a whole-mount section. A cancer focus was considered as spatially separate or multifocal if it was $3 \mathrm{~mm}$ or more from the closest cancer in any single section or $4 \mathrm{~mm}$ or more from the closest cancer on the adjacent section above or below, as described previously. ${ }^{17}$ The largest tumor focus was designated as the index nodule, and additional smaller tumors were labeled as multifocal tumors. The index nodule showed the highest Gleason score in the majority of cases. In rare cases where a smaller multifocal tumor had higher Gleason scores compared with the index tumor, the smaller multifocal tumor focus with the highest Gleason scores was considered as the index nodule. For each prostatectomy, the total number of tumor foci, linear dimension of index nodule, total linear tumor dimension, and Gleason scores of all tumor foci were documented. At the University of Michigan Health System, the greatest linear dimension of the index tumor rather than index tumor volume is reported clinically. Hence, we used the summed greatest linear dimensions of all tumor foci (total tumor linear dimension) as a measurement of cancer volume. Adjacent high-grade prostatic intraepithelial neoplasia was defined as high-grade prostatic intraepithelial neoplasia $\leq 4 \mathrm{~mm}$ from a focus of prostate cancer; the presence of adjacent high-grade prostatic intraepithelial neoplasia was noted for all cancer foci. Isolated high-grade prostatic intraepithelial neoplasia was defined as high-grade prostatic intraepithelial neoplasia $>4 \mathrm{~mm}$ from a focus of prostate cancer and was assessed in 15 randomly selected cases.

Immunohistochemistry for ERG (see below) was previously performed on sections representing all index and multifocal tumor foci from each case. All tumor foci were assigned as ERG positive or ERG negative. The total linear dimension of ERG-positive cancer summed the total linear size of the largest dimension of all ERG-positive tumor foci, including the index tumor when ERG was positive. ${ }^{12}$

PCA3 RNA in situ hybridization was performed on all sections representing all index and multifocal tumor foci from each case. PCA3 score (see below) was assigned based on the area of strongest staining in a given tumor focus. $P C A 3$ expression was considered as focal if $<50 \%$ of tumor cells in a given tumor focus were PCA3 positive. The total $P C A 3$-positive linear tumor dimension summed the total linear size of the largest dimension of all PCA3positive tumor foci in each case. Total PCA3 intensity was assigned as the sum of $P C A 3$ intensity scores of all tumor foci per case. Because PCA3 expression is scored based on a ranking system ranging from 0 to 4, PCA3-positive tumor volume was additionally scored using PCA3 product score (summed linear dimension of each tumor focus multiplied by its PCA3 intensity score). 


\section{ERG Immunohistochemistry}

Immunohistochemistry on unstained formalinfixed, paraffin-embedded levels of all tumor foci from the prostatectomy specimen blocks was performed using a monoclonal antibody against ERG, clone EPR 3864 (Epitomics, Burlingame, CA), using the automated Discovery XT staining platform (Ventana Medical Systems-A Roche group, Tucson, AZ, USA) and evaluated by the study pathologists JIW and LPK. ${ }^{12}$ Staining of vessels was used as a positive control and slides without staining of vessels were excluded from further analysis.

\section{RNA In Situ Hybridization}

Formalin-fixed paraffin-embedded sections were baked at $60{ }^{\circ} \mathrm{C}$ for $1 \mathrm{~h}$. RNA in situ hybridization was performed using a PCA3 RNA probe (NR_015342 regions 1683-2816; http://www.ncbi. nlm.nih.gov/nuccore/nr_015342) and an RNAscope FFPE Reagent Kit 2.0 (Advanced Cell Diagnostics, Hayward, CA) according to the manufacturer's instructions. Briefly, tissues were deparaffinized by immersing in xylene twice for $15 \mathrm{~min}$ each with periodic agitation. The slides were then immersed in $100 \%$ ethanol twice for 3 min each with periodic agitation then air-dried for $5 \mathrm{~min}$. Tissues were circled using a pap pen (Vector, \#H-4000), allowed to dry and treated with Pretreatment 1 buffer for $10 \mathrm{~min}$. Slides were rinsed in deionized water and then boiled in 1X Pretreatment 2 buffer for $15 \mathrm{~min}$. Slides were rinsed again in deionized water and then treated with Pretreatment 3 buffer for 30 min at $40{ }^{\circ} \mathrm{C}$ in a humidity chamber. Slides were rinsed twice in deionized water and then incubated with target probes for $P C A 3$ and $\mathrm{DapB}$, a bacterial gene used as a negative control, for $2 \mathrm{~h}$ at $40^{\circ} \mathrm{C}$ in a humidity chamber. Slides were then washed in $1 \mathrm{X}$ Wash Buffer twice for 2 min. Slides were then treated with Amp 1 solution for $30 \mathrm{~min}$, Amp 2 solution for $15 \mathrm{~min}$, Amp 3 solution for $30 \mathrm{~min}$, and Amp 4 solution for $15 \mathrm{~min}$, all at $40^{\circ} \mathrm{C}$ in a humidity chamber with two washes in $1 \mathrm{X}$ Wash Buffer for 2 min after each step. Slides were then treated with Amp 5 solution for $30 \mathrm{~min}$ and Amp 6 solution for $15 \mathrm{~min}$ at room temperature in a humidity chamber with two washes in $1 \mathrm{X}$ Wash Buffer for 2 min after each step. Color was developed by adding a 1:60 solution of Fast Red B:Fast Red A to each slide and incubated for $10 \mathrm{~min}$. Slides were washed twice in deionized water and then immersed in a 50\% hematoxylin (Fisher, \#SH26-4D) solution for $2 \mathrm{~min}$. Slides were rinsed several times in deionized water and then immersed in a $0.01 \%$ ammonium hydroxide solution. Slides were rinsed in deionized water then dehydrated by immersing five times in $70 \%$ ethanol twice, immersing five times in $95 \%$ ethanol twice, immersing twice in 100\% ethanol for $5 \mathrm{~s}$ each and in xylene for $5 \mathrm{~s}$. The slides were mounted in Cytoseal XYL (Thermo Scientific, \#8312-4) for viewing under bright-field microscope. Positive controls were performed for all runs using a POLR2A gene-specific RNA probe. PCA3 RNA-ISH was not repeated in any tumor focus showing a negative result to avoid discrepant analysis.

\section{RNA In Situ Hybridization Evaluation Criteria}

RNA in situ hybridization expression intensity scoring guidelines were established to classify tumor foci as PCA3 positive or PCA3 negative. PCA3 expression by RNA in situ hybridization appeared as distinct cytoplasmic punctate dots. All tumor foci were evaluated and scanned at $\times 20$ magnification. Scoring for an entire tumor focus was based on the highest PCA3 intensity using these criteria. On the basis of the number of dots/cell, we established five grading levels ranging from 0 to 4 : tumor foci with no staining or $<1 \mathrm{dot} / \mathrm{cell}$ at $\times 20$ magnification were scored as zero; foci with 1-3 dots/cell in $>5 \%$ of the tumor were scored as 1 ; tumor foci with 4-10 dots/cell with no or very few dot clusters (fused overlapping dots) in $>5 \%$ of tumor were scored as 2; tumor foci with $>10$ dots/ cell with $<10 \%$ of positive cells having dot clusters in $>5 \%$ of tumor were scored as 3 ; and tumor foci with $>10$ dots/cell with $>10 \%$ of positive cells having dot clusters in $>5 \%$ of tumor were scored as 4 (Figure 1). Expression was considered as focal when dots were seen in $<50 \%$ of cells in a tumor focus. For the purposes of this study, tumor foci showing scores 2-4 were considered as PCA3 positive, whereas tumor foci showing scores 0-1 were considered as $P C A 3$ negative. All PCA3 slides were reviewed by study pathologists LPK and JIW.

\section{Tissue PCA3 Expression by In Situ Hybridization vs Transcription-Mediated Amplification}

Because urine PCA expression by transcriptionmediated amplification was compared with tissue PCA3 expression by in situ hybridization, tissue $P C A 3$ expression values by in situ hybridization were compared with tissue values by transcriptionmediated amplification. Nine tissue blocks were selected, on which PCA3 RNA in situ hybridization had been performed-4 blocks demonstrating prostate cancer with expression intensity of 4 (diffuse), and 5 blocks with expression intensity of 0 . Two 10 micron sections were taken from each block, changing the blade between blocks to prevent crosscontamination. Both sections from each block were placed in a specimen transport medium, heated in a $60{ }^{\circ} \mathrm{C}$ water bath for $30 \mathrm{~min}$ with occasional swirling, then placed on ice for $5 \mathrm{~min}$. Separate sterile swabs were used to remove solidified and floating paraffin. Specimen tubes were then capped, mixed by inverting five times, and stored at $-70^{\circ} \mathrm{C}$ until testing. Before analysis, samples were warmed to $60{ }^{\circ} \mathrm{C}$ in a water bath for $<20 \mathrm{~min}$. $P C A 3$ scores were 


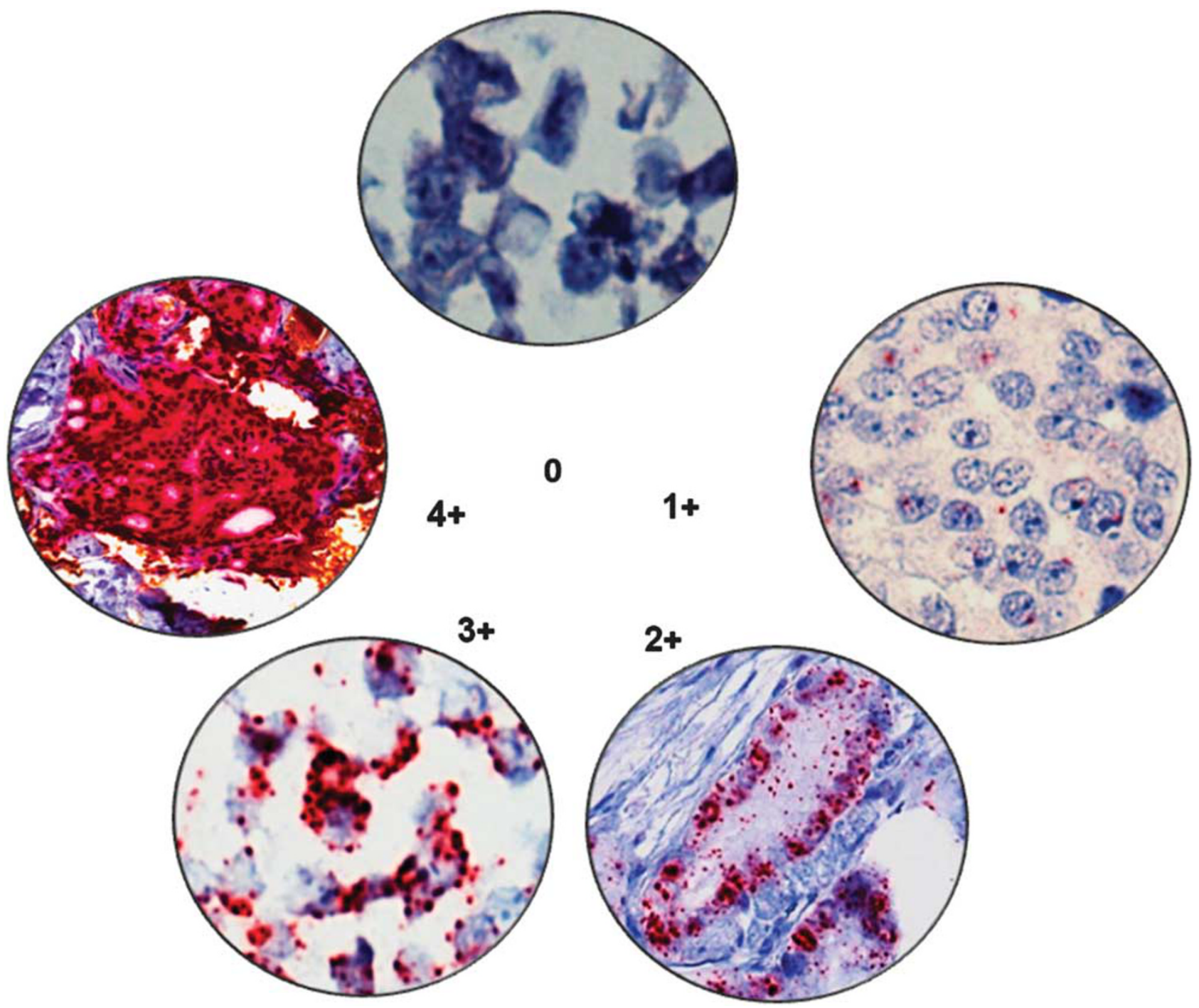

Figure 1 PCA3 intensity scores; tumor foci with no staining or $<1$ dot/cell at $\times 40$ magnification were scored as zero; foci with 1-3 dots/ cell in $>5 \%$ of the tumor were scored as 1 ; tumor foci with $4-10$ dots/cell with no or very few dot clusters (fused overlapping dots) in $>5 \%$ of the tumor were scored as 2 ; tumor foci with $>10$ dots/cell with $<10 \%$ of positive cells having dot clusters in $>5 \%$ of the tumor were scored as 3; tumor foci with $>10$ dots/cell with $>10 \%$ of positive cells have dot clusters in $>5 \%$ of the tumor were scored as 4 .

determined by transcription-mediated amplification, as previously described. ${ }^{13}$

\section{Statistical Analysis}

All statistical analyses were performed using the Stats package in the $\mathrm{R}$ programming language. The two-tailed $t$-test was used to analyze tumor focus size vs PCA3 status, and PCA3-positive tumor size in urine $P C A 3$-high vs PCA3-low groups. Fisher's exact test was used to analyze ERG status vs PCA3 status of tumor foci and, PCA3 status of tumor foci vs PCA3 status of adjacent high-grade prostatic intraepithelial neoplasia. Spearman $\rho\left(r_{\mathrm{S}}\right)$ was used to test associations between urine $P C A 3$ and total tumor linear dimension, total number of tumor foci, total PCA3 intensity, PCA3-positive tumor linear dimension, and index nodule PCA3 score. Spearman $\rho\left(r_{\mathrm{s}}\right)$ was also used to evaluate correlations between urine TMPRSS2-ERG and number of PCA3positive tumor foci, urine TMPRSS2-ERG, number of ERG-positive tumor foci, number of PCA3-positive tumor foci, and number of ERG-positive tumor foci. Wilcoxon rank-sum test was used to analyze total $P C A 3$ intensity, and PCA3 product score, in urine PCA3-high vs urine PCA3-low groups.

\section{Results}

\section{Prostatectomy Cohort}

As previously reported, the 41 prostatectomy specimens in this study had a median of 3 cancer foci (range 1-11) and median total linear dimension of $2.5 \mathrm{~cm}$ (range $0.4-5.5 \mathrm{~cm}$ ). ${ }^{12}$ The majority of cases were confined to the prostate (pT2, 37/41, 90\%) and had an index tumor Gleason score of 7 (31/41, 76\%). 


\section{PCA3 Expression by ISH and Correlation with ERG Expression by IHC}

A total of 138 tumor foci were evaluated with $P C A 3$ RNA in situ hybridization. Of the 159 tumor foci identified in our previous study, ${ }^{12} 21$ small tumor foci were lost on deeper sectioning. Of the 138 tumor foci evaluated, 77 (56\%) were PCA3 positive. PCA3 intensities for all tumor foci were 4 (43 foci, $31 \%$ ), 3 (29 foci, 21\%), 2 (5 foci, 4\%), 1 (20 foci, $14 \%$ ), and 0 (41 foci, $30 \%$ ). The index tumor was PCA3 positive in 25 cases (61\%). PCA3 intensities for index tumor nodules were 4 (16 tumor nodules, 39\%), 3 (9 nodules, 22\%), 2 (1 nodule, 2\%), 1 (6 nodules, $15 \%$ ), and 0 (9 nodules, $22 \%$ ). Of all PCA3positive cancer foci, 29 (37\%) showed focal PCA3 staining. PCA3 expression was noted in at least one tumor focus in 36 cases (88\%). PCA3 status of index tumor foci did not associate with the Gleason score $(P=0.34$, Spearman correlation). The pathologic data, PCA3 status, and urine PCA3 scores are summarized in Table 1. Microphotographs demonstrating diffuse and focal PCA3 expression are present in Figure 2 .

The median total PCA3-positive linear tumor dimension in the 41 patients was $1.6 \mathrm{~cm}$ (range $0-5.1 \mathrm{~cm})$. The median number of PCA3-positive tumor foci per case was 2 (range 0-7). The median total PCA3 intensity across all 41 cases was 7 (range $0-26$ ). The median PCA3 product score was 6 (range 0-20). PCA3-positive tumor foci were overall larger than PCA3-negative tumor foci (median 0.9 VS $0.6 \mathrm{~cm}$, respectively; two-tailed $t$-test, $P<0.01$ ).

ERG expression was identified in 70 tumor foci (50\%), and 53 tumor foci (38\%) were positive for both ERG and PCA3. Overall, across all tumor foci, 94 foci $(68 \%)$ were positive for ERG, PCA3, or both (Figure 3). There was a significant positive association between PCA3 status by RNA in situ hybridization and ERG status by immunohistochemistry in tumor foci $(P<0.01$, Fisher's exact test). The median PCA3 intensity was $3+$ in ERG-positive nodules and $1+$ in ERG-negative nodules. Of the 41 cases, $95 \%(39 / 41)$ had at least one tumor focus positive for either PCA3 by RNA in situ hybridization or ERG expression by immunohistochemistry.

\section{PCA3 Expression in High-Grade Prostatic Intraepithelial Neoplasia}

We identified 66 foci of high-grade prostatic intraepithelial neoplasia adjacent to cancer, of which $51(77 \%)$ were $P C A 3$ positive. Tissue PCA3 intensities for adjacent high-grade prostatic intraepithelial neoplasia were $4(29,44 \%), 3(19,29 \%), 2$ $(3,5 \%), 1(4,6 \%)$, and $0(11,17 \%)$. High-grade prostatic intraepithelial neoplasia adjacent to $P C A 3-$ positive cancer tended to be $P C A 3$ positive $(90 \%$, $38 / 42$ foci). In contrast, high-grade prostatic intraepithelial neoplasia adjacent to PCA3-negative cancer was nearly equally divided into $P C A 3$ positive
(54\%; 13/24 tumor foci) and PCA3 negative (46\%; $11 / 24$ tumor foci) (Figures 4 and 5).

We found 16 foci of isolated high-grade prostatic intraepithelial neoplasia after careful review of 15 randomly selected cases. Of these, 56\% (9/16) were PCA3 positive by RNA-ISH. PCA3 scores for isolated high-grade prostatic intraepithelial neoplasia were as follows: 4 (4, 25\%), $3(3,19 \%), 2(2,13 \%), 1$ (3, $19 \%)$, and $0(4,25 \%)$. In total, $71 \%(60 / 84)$ of all identified high-grade prostatic intraepithelial neoplasia foci were PCA3 positive.

All benign glands were examined for PCA3 expression. Of the benign glands, 50-60 acini (on 10 slides from 9 cases; intensity range 2-3) were $P C A 3$ positive. These glands were frequently seen close to ( $<3 \mathrm{~mm}$ from) PCA3-positive tumor foci and the remaining benign prostatic glands, including all foci of atrophy and adenosis, were PCA3 negative. Thus, across 136 tissue sections, positive PCA3 expression by RNA in situ hybridization showed a specificity of $>90 \%$ for combined prostate cancer and high-grade prostatic intraepithelial neoplasia.

\section{Urine PCA3 and Urine TMPRSS2-ERG}

All 41 patients had sufficient urine RNA for evaluation of PCA3 and TMPRSS2-ERG. Median urine PCA3 score was 40 (s.d. 38, range 3-186). Of these, $24(59 \%)$ had urine PCA3 scores $>35$, a proposed optimal cutoff for detection of cancer on biopsy, ${ }^{18}$ and 29 (71\%) had urine PCA3 score $>25$, the current Food and Drug Administration approved cutoff for predicting prostate cancer after a negative biopsy. Urine PCA3 score correlated well with number of tumor foci $\left(r_{\mathrm{s}}=0.51, P<0.01\right)$ and correlated weakly with summed total linear tumor dimension $\left(r_{\mathrm{s}}=0.29\right.$, $P=0.06$ ), consistent with our previous study using this cohort. $^{12}$ Urine PCA3 did not correlate significantly with the Gleason score of index tumor nodule ( $P=0.89$, Spearman correlation).

Urine PCA3 score did not correlate well with any independent measure of PCA3-positive tumor volume, including index nodule PCA3 score $\left(r_{\mathrm{s}}=-0.1, \quad P=0.52\right)$, total $P C A 3$-positive linear tumor dimension $\left(r_{\mathrm{s}}=0.09, P=0.56\right)$, or $P C A 3$ product score $\left(r_{\mathrm{s}}=0.04, P=0.78\right)$. Although urine $P C A 3$ did correlate with the number of PCA3positive tumor foci per case $\left(r_{\mathrm{s}}=0.34, P=0.03\right)$, this correlation became non-significant on multivariate linear regression including total number of tumor foci (outcome variable urine PCA3 score; total number tumor foci $P<0.01$, total number $P C A 3-$ positive tumor foci $P=0.88)$. Similarly, although total PCA3 intensity showed correlation with urine $P C A 3$ that approached statistical significance ( $r_{\mathrm{s}}=0.29, P=0.06$ ), this too was statistically nonsignificant on multivariate analysis (outcome variable urine PCA3 score; total number tumor foci $P<0.01$, total $P C A 3$ intensity $P=0.38$ ). Using both 35 and 25 as cutoff values for urine $P C A 3$, urine 
Table 1 Pathologic data and PCA3 scores for all cases

\begin{tabular}{|c|c|c|c|c|c|c|c|c|c|}
\hline $\begin{array}{l}\text { Case } \\
\text { number }\end{array}$ & $\begin{array}{c}\text { Summed } \\
\text { tumor } \\
\text { dimension } \\
(\mathrm{cm})\end{array}$ & $\begin{array}{l}\text { Summed PCA3+ } \\
\text { tumor dimension } \\
(\mathrm{cm})\end{array}$ & $\begin{array}{l}\text { Index nodule } \\
\text { dimension } \\
\text { (cm) }\end{array}$ & $\begin{array}{c}\text { Index nodule } \\
\text { PCA3 score } \\
\text { (0-4) }\end{array}$ & $\begin{array}{c}\text { Index } \\
\text { nodule } \\
\text { ERG status }\end{array}$ & $\begin{array}{l}\text { Index } \\
\text { nodule } \\
\text { Gleason } \\
\text { score }\end{array}$ & $\begin{array}{c}\text { Total } \\
\text { number of } \\
\text { cancer foci }\end{array}$ & $\begin{array}{l}\text { Total number of } \\
\text { PCA3 + cancer } \\
\text { foci }\end{array}$ & $\begin{array}{l}\text { Urine } \\
\text { PCA3 } \\
\text { score }\end{array}$ \\
\hline 1 & 2.2 & 0 & 1.2 & 0 & Negative & $3+3$ & 2 & 0 & 34.8 \\
\hline 2 & 1.6 & 0.1 & 1.4 & 0 & Negative & $3+4$ & 3 & 1 & 27 \\
\hline 3 & 3.5 & 3.5 & 1.8 & 4 & Negative & $4+3$ & 2 & 2 & 2.6 \\
\hline 4 & 1.2 & 0.1 & 0.8 & 0 & Negative & $3+3$ & 3 & 1 & 66.3 \\
\hline 5 & 2 & 2 & 1 & 3 & Negative & $\begin{array}{c}3+4 \\
\text { (tertiary 5) }\end{array}$ & 2 & 2 & 59.6 \\
\hline 6 & 2.8 & 2.3 & 1.5 & 4 & Negative & $4+3$ & 3 & 2 & 19.1 \\
\hline 7 & 1.7 & 1 & 0.7 & 3 & Negative & $3+4$ & 3 & 2 & 73.9 \\
\hline 8 & 1 & 0 & 0.6 & 1 & Negative & $3+4$ & 2 & 0 & 49.7 \\
\hline 9 & 0.4 & 0.2 & 0.2 & 1 & Negative & $3+3$ & 2 & 1 & 12.6 \\
\hline 10 & 3.4 & 0.6 & 2.8 & 1 & Negative & $\begin{array}{c}4+3 \\
\text { (tertiary 5) }\end{array}$ & 3 & 2 & 74 \\
\hline 11 & 2.1 & 2 & 2 & 4 & Negative & $3+4$ & 2 & 1 & 42.4 \\
\hline 12 & 2.3 & 2.1 & 1.9 & 4 & Negative & $4+3$ & 3 & 2 & 86.3 \\
\hline 13 & 1.7 & 0.1 & 0.7 & 0 & Negative & $3+3$ & 6 & 1 & 22 \\
\hline 14 & 0.6 & 0 & 0.6 & 0 & Positive & $4+4$ & 1 & 0 & 3.2 \\
\hline 15 & 0.9 & 0 & 0.6 & 0 & Positive & $3+3$ & 3 & 0 & 82.9 \\
\hline 16 & 1.8 & 0.9 & 0.9 & 1 & Negative & $3+4$ & 2 & 1 & 15.3 \\
\hline 17 & 2.3 & 0.6 & 0.7 & 0 & Negative & $3+4$ & 6 & 2 & 71.8 \\
\hline 18 & 4.5 & 1.6 & 1.6 & 3 & Negative & $3+4$ & 5 & 1 & 54.9 \\
\hline 19 & 2.2 & 1.7 & 1.1 & 4 & Positive & $3+4$ & 3 & 2 & 6.3 \\
\hline 20 & 1.1 & 1.1 & 1.1 & 4 & Positive & $3+4$ & 1 & 1 & 7.6 \\
\hline 21 & 1.3 & 1.2 & 1.1 & 4 & Positive & $3+4$ & 3 & 2 & 38.3 \\
\hline 22 & 1.3 & 1.2 & 1.2 & 3 & Positive & $3+4$ & 2 & 1 & 12.4 \\
\hline 23 & 1.3 & 0 & 1.3 & 0 & Positive & $3+4$ & 1 & 0 & 31.2 \\
\hline 24 & 3.4 & 3.4 & 1.6 & 4 & Negative & $3+4$ & 4 & 4 & 16.9 \\
\hline 25 & 3 & 1.5 & 0.8 & 3 & Positive & $3+4$ & 4 & 2 & 104.5 \\
\hline 26 & 2.3 & 1.9 & 1 & 4 & Positive & $3+3$ & 6 & 3 & 95.2 \\
\hline 27 & 3.2 & 2.1 & 2.1 & 4 & Positive & $4+3$ & 2 & 1 & 35.8 \\
\hline 28 & 2.9 & 2.6 & 1 & 3 & Positive & $3+3$ & 4 & 3 & 37 \\
\hline 29 & 2.7 & 1.1 & 1.6 & 0 & Negative & $3+4$ & 3 & 2 & 125.9 \\
\hline 30 & 3.1 & 2.6 & 1.9 & 4 & Positive & $3+4$ & 3 & 2 & 32 \\
\hline 31 & 5.1 & 5.1 & 2 & 4 & Positive & $3+4$ & 6 & 6 & 74.5 \\
\hline 32 & 2.7 & 2.1 & 2.1 & 4 & Positive & $3+4$ & 2 & 1 & 10.5 \\
\hline 33 & 5.5 & 4.2 & 1.5 & 3 & Positive & $3+4$ & 4 & 3 & 46.9 \\
\hline 34 & 2.6 & 1.6 & 1 & 4 & Positive & $3+4$ & 5 & 3 & 32.9 \\
\hline 35 & 4.9 & 2.8 & 1.6 & 3 & Positive & $3+4$ & 5 & 2 & 68.9 \\
\hline 36 & 3.5 & 3.5 & 2.4 & 4 & Positive & $3+4$ & 3 & 3 & 41.6 \\
\hline 37 & 3.4 & 1.4 & 2 & 1 & Positive & $3+4$ & 3 & 2 & 10.6 \\
\hline 38 & 4.9 & 4.9 & 1.4 & 3 & Positive & $3+3$ & 7 & 7 & 43.2 \\
\hline 39 & 3.8 & 3.8 & 1.9 & 4 & Positive & $3+4$ & 3 & 3 & 105.1 \\
\hline 40 & 5.3 & 1.6 & 0.8 & 1 & Positive & $3+4$ & 11 & 3 & 186.3 \\
\hline 41 & 5.1 & 2.1 & 2.1 & 2 & Positive & $3+4$ & 3 & 1 & 39.6 \\
\hline
\end{tabular}

PCA3-high and PCA3-low groups showed no statistically significant difference in $P C A 3$-positive tumor linear dimension $(P=0.09$ for cutoff $35, P=0.46$ for cutoff 25; two-tailed $t$-test) or $P C A 3$ product score $(P=0.21$ for cutoff $35, P=0.99$ for cutoff 25 ; Wilcoxon rank-sum test) (Table 2).

In our cohort, five patients $(12 \%)$ had no PCA3positive tumor focus by RNA in situ hybridization (total nine tumor foci). The median urine PCA3 score in these patients was 34.8 (range 3.2-82.9). Similarly, two patients (5\%) showed no PCA3-positive focus by RNA in situ hybridization or ERG-positive focus by immunohistochemistry. Urine PCA3 scores in these patients were 34.8 and 49.7 .

Of the 41 patients, 12 (29\%) had urine $P C A 3<25$. Of these, $92 \%(11 / 12)$ had at least one $P C A 3$-positive tumor focus. Median number of PCA3-positive tumor foci was 1 (range 0-4). Median
PCA3-positive linear tumor dimension was $1.3 \mathrm{~cm}$ (range 0-3.5). PCA3 intensities of index tumor nodules in these patients were as follows: 4 (6 nodules, 50\%), 3 (1 nodule, 8\%), 1 (3 nodules, $25 \%$ ), and 0 ( 2 nodules, $17 \%$ ). Of the seven index nodules with PCA3 intensity 3 or 4 , three of them demonstrated diffuse expression within the tumor focus. Median urine $P C A 3$ score in these seven patients with index nodule showing PCA3 intensity 3 or 4 was 10.5 (range 2.3-19.1). Five patients with urine $P C A 3$ values $<25$ had total PCA3-positive linear dimension $>1.6 \mathrm{~cm}$ (the median value).

Urine TMPRSS2-ERG score correlated with number of PCA3-positive tumor foci $\left(r_{\mathrm{s}}=0.34, P=0.03\right)$. However, number of ERG-positive tumor foci correlated much more strongly with urine TMPRSS2:ERG score $\left(r_{\mathrm{s}}=0.64, P<0.01\right)$, as shown in our previous study, ${ }^{12}$ and number of ERG-positive tumor foci 


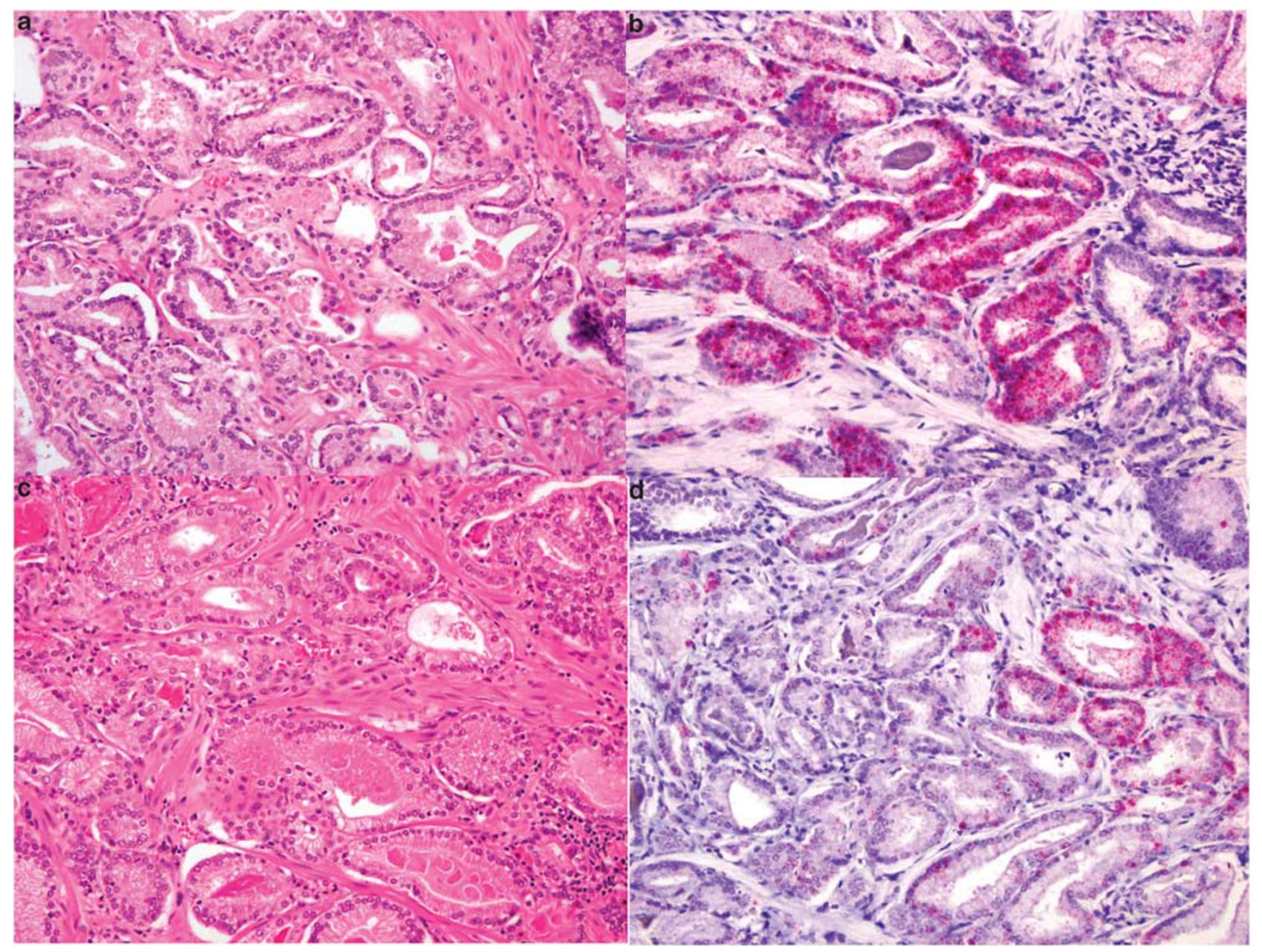

Figure 2 PCA3 (RNA in-situ hydridization) expression with the corresponding H\&E-stained sections. Diffuse expression with maximum intensity score 4 (a, H\&E; b, PCA3 RNA in situ hybridization; both at $\times 200$ magnification). Focal expression with intensity score 4 at right side of image and 0 at left side of image (c, H\&E; d, PCA3 RNA in-situ hybridization; both at $\times 200$ magnification).

correlated strongly with number of $P C A 3$-positive tumor foci $\left(r_{\mathrm{s}}=0.63, P<0.01\right)$.

\section{Tissue PCA3 Expression by In Situ Hybridization vs Transcription-Mediated Amplification}

The four tumor foci that were strongly PCA3 positive by RNA in situ hybridization (4/4 intensity) had a median tissue PCA3 score of 10 (range 10-19) by transcription-mediated amplification performed on tissue. Conversely, the five tumors that were PCA3 negative by RNA in situ hybridization $(0 / 4$ intensity) showed a median tissue $P C A 3$ score of 3 (range 2-4) by transcription-mediated amplification. These differences in PCA3 score were statistically significant (Wilcoxon rank-sum test, $P=0.02$ ). See Table 3 for details.

\section{Discussion}

$P C A 3$, a non-coding RNA, was originally reported as specifically overexpressed in prostate cancer by

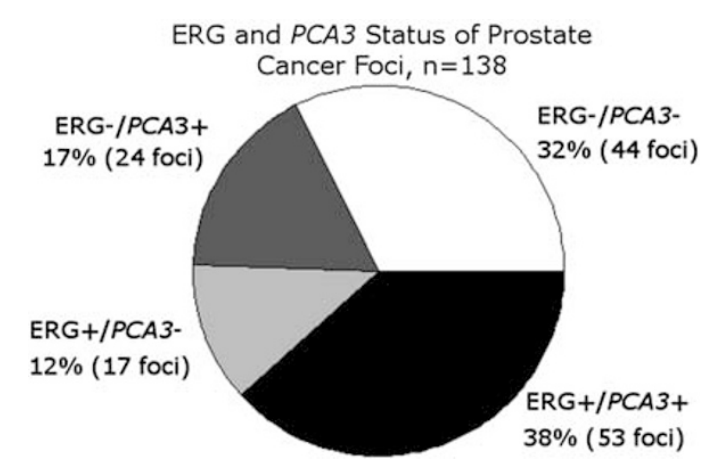

Figure 3 PCA3 RNA in situ hybridization and ERG immunohistochemistry status of all tumor foci.

Bussemaker et al. ${ }^{1}$ In the present study, using a novel RNA in situ hybridization technique to evaluate $P C A 3$ expression in prostate cancer, we have shown that PCA3 RNA is significantly elevated in prostate cancer tissue relative to benign prostatic tissue. This is the first time that PCA3 has been shown to display a strong specificity $(>90 \%)$ for cancer and high-grade prostatic intraepithelial 


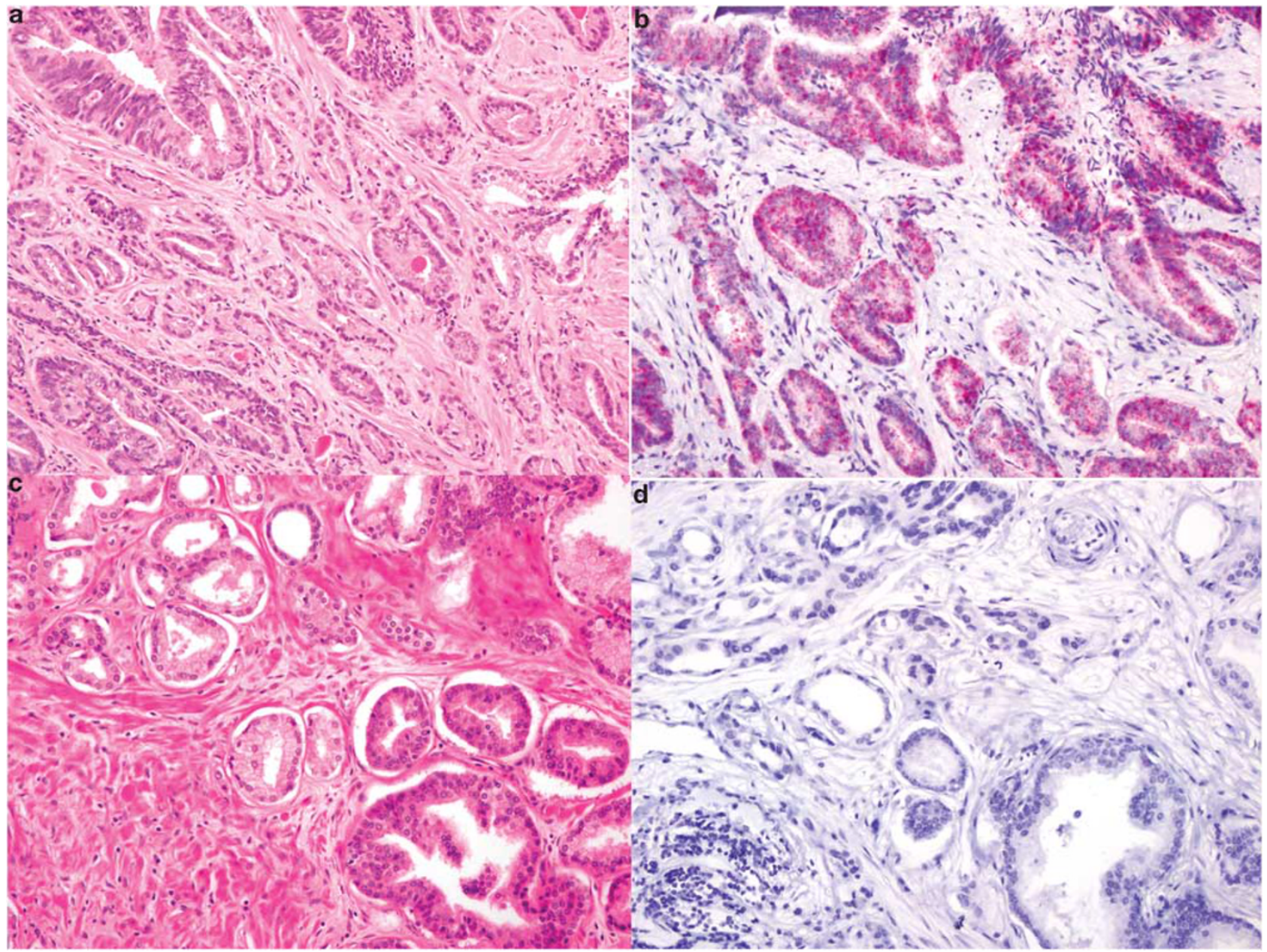

Figure 4 PCA3 RNA in situ hybridization in prostate cancer and adjacent high-grade prostatic intraepithelial neoplasia showing concordant positive (a, H\&E; b, PCA3 RNA in situ hybridization; both at $\times 200$ magnification) and negative (c, H\&E; d, PCA3 RNA in situ hybridization; both at $\times 200$ magnification) PCA3 status.

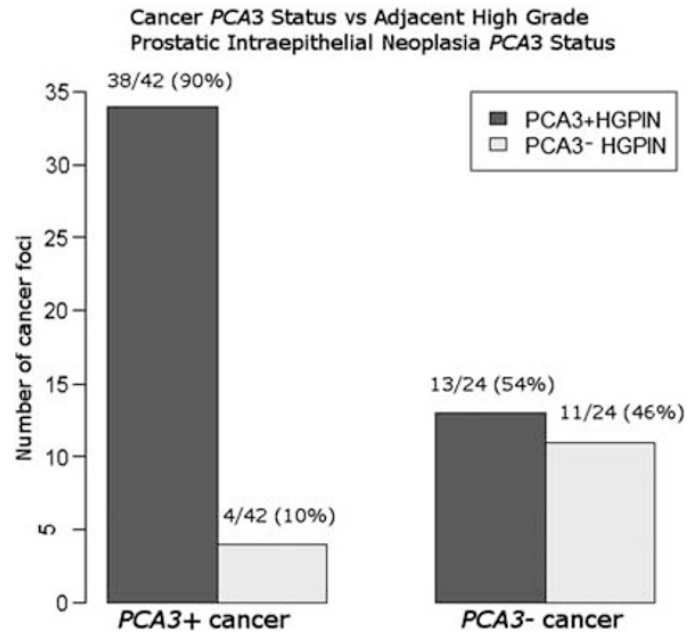

Figure 5 PCA3 RNA in situ hybridization status of prostate cancer and adjacent high-grade prostatic intraepithelial neoplasia.

neoplasia in formalin-fixed paraffin-embedded radical prostatectomy specimens. In this study, we systematically mapped all the tumor foci, and found
$88 \%$ of all cases in our cohort showed at least one PCA3-positive tumor focus. The results of the current study are consistent with previous tissue studies using PCR methods, which showed that although benign prostatic tissue displays low-level $P C A 3$ expression, expression in prostate cancer tissue is considerably higher in comparison with benign tissue. ${ }^{1}$

Due to the inability to develop an immunohistochemistry-based detection platform for $P C A 3$, there have been very few studies of PCA3 expression in tissue. To our knowledge, a study by Popa et $a l^{3}$ is the only other study evaluating PCA3 expression in prostate cancer tissue using in situ hybridization. In a series of 24 and 26 prostate cancers evaluated with chromogenic and radioactive in situ hybridization, respectively, their group demonstrated $P C A 3$ expression in the majority of prostate cancer $(92-96 \%)$ and at least focal cytoplasmic expression in the majority of high-grade prostatic intraepithelial neoplasia (71-96\%). PCA3 expression was also noted in almost a third of benign glands $(29-33 \%)$, showing at least focal staining, and 
Table 2 Fraction of cases positive for urine PCA3 at cutoffs $>25$ and $>35$, by total tumor linear dimension (cm) and total PCA3 + tumor linear dimension $(\mathrm{cm})$

\begin{tabular}{|c|c|c|c|c|c|c|c|c|}
\hline & \multicolumn{4}{|c|}{$\begin{array}{l}\text { Summed linear tumor } \\
\text { dimension }(\mathrm{cm})\end{array}$} & \multicolumn{4}{|c|}{$\begin{array}{l}\text { Summed PCA3 + linear } \\
\text { tumor dimension }(\mathrm{cm})\end{array}$} \\
\hline & $0-1$ & $1.1-2.5$ & $2.6-3.5$ & $>3.5$ & $0-1$ & $1.1-2.5$ & $2.6-3.5$ & $>3.5$ \\
\hline Urine $P C A 3$ score $>35$ & $0 / 4(0 \%)$ & $8 / 16(50 \%)$ & $6 / 8(75 \%)$ & $8 / 13(62 \%)$ & $6 / 13(46 \%)$ & $11 / 18(61 \%)$ & $3 / 6(50 \%)$ & $4 / 4(100 \%)$ \\
\hline Urine $P C A 3$ score $>25$ & $2 / 4(50 \%)$ & $11 / 16(69 \%)$ & $8 / 8(100 \%)$ & $8 / 13(62 \%)$ & $9 / 13(69 \%)$ & $12 / 18(67 \%)$ & $4 / 6(67 \%)$ & $4 / 4(100 \%)$ \\
\hline Urine $P C A 3$ score $<25$ & $2 / 4(50 \%)$ & $5 / 16(31 \%)$ & $0 / 8(0 \%)$ & $5 / 13(38 \%)$ & $4 / 13(31 \%)$ & $6 / 18(33 \%)$ & $2 / 6(33 \%)$ & $0 / 4(0 \%)$ \\
\hline
\end{tabular}

indicating poor specificity for prostate cancer. Some differences between our study and that of Popa et al bear mentioning. In the Popa et al study, the percentage of cells expressing PCA3 was evaluated, and PCA3 expression was reported as positive or negative. The presence of a single positive cell was defined as positive. In contrast, we used a commercially available well-standardized RNA in situ detection procedure for bright-field application. In this method, oligonucleotide-based RNA probes are designed to yield punctate dots for each RNA transcript for a semiquantitative evaluation of tissue level expression of PCA3 RNA. Based on this, we were able to compare differences in the level of PCA3 RNA expression among tumor foci. Therefore, we are the first to introduce the development of evaluation criteria for PCA3 in formalin-fixed and paraffin-embedded tissue. The present study is also considerably larger, encompassing a well-characterized cohort of 138 tumor foci in radical prostatectomy specimens, in contrast to the 48 tissue blocks (28 prostatic cancers and 20 benign prostatic tissues) utilized in the previous study. Also, in contrast to the study by Popa et al, the present study used a non-radioactive method, thereby proposing a more clinically realistic assay.

Recurrent TMPRSS2-ERG fusions present in $\sim 50 \%$ of PSA-detected prostate cancers result in overexpression of ERG protein product. ${ }^{4,8-10,19}$ The fusion is reliably detectable using fluorescence in situ hybridization, ${ }^{6,8,9}$ and immunohistochemistry using monoclonal antibodies directed against ERG has shown high concordance with fluorescence in situ hybridization results. ${ }^{10,11,19-21}$ We and others have shown that ERG IHC is $>99 \%$ specific for prostate cancer and high-grade prostatic intraepithelial neoplasia, showing only rare expression in benign prostatic glands. ${ }^{10,11}$ The present study showed $55 \%$ of cancer foci were PCA3 positive by RNA in situ hybridization, a fraction slightly higher than that seen for the sensitivity of ERG immunohistochemistry in the present cohort $(50 \%)$ and previous studies. ${ }^{8,10}$ Similarly, while $88 \%$ of cases had at least one PCA3-positive cancer focus, slightly fewer $(76 \%)$ had at least one ERG-positive cancer focus. We found a strong, statistically significant positive association $(P<0.01)$ between ERG status by immunohistochemistry and PCA3 status by RNA in situ
Table $3 P C A 3$ and PSA RNA values in copies/ml, as measured by transcription-mediated amplification

\begin{tabular}{lcccc}
\hline & $\begin{array}{c}\text { PCA3 } \\
R N A \\
\text { Case number }\end{array}$ & $\begin{array}{c}\text { PSA } \\
\text { mRNA } \\
\text { value }\end{array}$ & $\begin{array}{c}\text { PCA3: } \\
\text { PSA } \\
\text { ratio }^{\mathrm{b}}\end{array}$ & $\begin{array}{c}\text { PCA3 In situ } \\
\text { hybridization } \\
\text { intensity }\end{array}$ \\
\hline 12-index tumor & 18458 & 1870578 & 10 & 4 \\
30-index tumor & 32681 & 1694140 & 19 & 4 \\
32-index tumor & 22178 & 2199705 & 10 & 4 \\
36-index tumor & 20544 & 2093452 & 10 & 4 \\
1-index tumor & 2360 & 556125 & 4 & 0 \\
17-index tumor & 5932 & 2539530 & 2 & 0 \\
30-non-index tumor & 2364 & 920239 & 3 & 0 \\
41-non-index tumor & 4282 & 1542647 & 3 & 0 \\
41-non-index tumor & 3977 & 1167430 & 3 & 0 \\
\hline
\end{tabular}

${ }^{\mathrm{a}}$ Case number refers to numbering in Table 1.

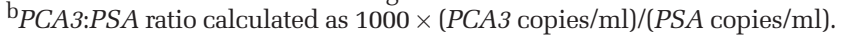

hybridization in prostate cancer foci. To the best of our knowledge, this is the first report of such a positive association. A subset of tumor foci (44/138 $(32 \%))$ were completely negative for both markers. Despite the strong association between PCA3 and ERG expression in tumor foci, a subset of tumor foci (41, 29\%) also showed inverse ERG and PCA3 status. Consistent with these findings, $68 \%$ of tumor foci were positive for either PCA3 or ERG.

High-grade prostatic intraepithelial neoplasia has displayed molecular abnormalities similar to prostate cancer in numerous studies, including chromosomal losses and gains, ${ }^{22,23}$ telomere shortening, ${ }^{24}$ and AMACR overexpression. ${ }^{25}$ Consistent with those findings, $71 \%$ of high-grade prostatic intraepithelial neoplasia foci in the present study were $P C A 3$ positive. Interestingly, while the majority $(90 \%)$ of high-grade prostatic intraepithelial neoplasia foci adjacent to $P C A 3$-positive cancer was also PCA3 positive, slightly over half of isolated high-grade prostatic intraepithelial neoplasia (56\%) and high-grade prostatic intraepithelial neoplasia adjacent to PCA3-negative cancer (44\%) were PCA3 positive. The relationship between ERG status of high-grade prostatic intraepithelial neoplasia and associated prostate cancer differs from that of $P C A 3$, in that ERG-positive high-grade prostatic intraepithelial neoplasia has been shown to be strongly associated with adjacent ERG-positive prostate cancer. ${ }^{13,17}$ Furthermore, in contrast to the large fraction of high-grade prostatic intraepithelial neoplasia foci showing PCA3 expression (71\%) in 
the present study, several studies have shown that only a small fraction of high-grade prostatic intraepithelial neoplasia foci $(15-18 \%)$ demonstrate ERG expression. ${ }^{10,21}$ These findings favor the hypothesis that ERG-positive high-grade prostatic intraepithelial neoplasia may indicate unsampled prostate cancer or high-grade prostatic intraepithelial neoplasia that may progress to invasive carcinoma, which does not appear to be true of $P C A 3$-positive high-grade prostatic intraepithelial neoplasia. In summary, while ERG-positive highgrade prostatic intraepithelial neoplasia represents the minority of high-grade prostatic intraepithelial neoplasia and may be predictive of adjacent ERGpositive prostate cancer, PCA3-positive high-grade prostatic intraepithelial neoplasia appears to represent the majority of high-grade prostatic intraepithelial neoplasia and does not appear to be predictive of adjacent prostate cancer.

The great majority of clinical studies of $P C A 3$ have been on urine measurement, which has demonstrated superior sensitivity and specificity to serum PSA for the detection of clinically significant prostate cancer on biopsy. ${ }^{13,26-28}$ Similarly, TMPRSS2-ERG mRNA can be detected in the urine of patients with prostate cancer, and correlates with linear extent of ERG-positive cancer by immunohistochemistry. ${ }^{19,21}$ In the present study, urine PCA3 score did not correlate with any measure of PCA3positive tumor burden by RNA in situ hybridization except the number of $P C A 3$-positive tumor foci per case $\left(r_{\mathrm{s}}=0.34, P=0.03\right)$, which became nonsignificant on multivariate analysis including total number of tumor foci. In contrast, a strong correlation has been shown between urine TMPRSS2:ERG and both the total ERG-positive tumor dimension $\left(r_{\mathrm{s}}=0.68\right)$ and the number of ERG-positive tumor foci $\left(r_{\mathrm{s}}=0.67\right) .{ }^{12}$

Interestingly, $12 \%(5 / 41)$ of cases in the present study had no PCA3-positive tumor focus. Of these, $80 \%(4 / 5)$ had urine $P C A 3$ values $>25$, the current Food and Drug Administration approved the cutoff value. There are several possible explanations for this lack of correlation between urine and tissue PCA3 expression levels. First, while ERG immunohistochemistry shows strong diffuse expression within positive tumor foci, PCA3 RNA in situ hybridization tended to show focal expression, with variations in intensity within a given tumor focus. Thus, in contrast to ERG, in which quantification in tissue is relatively straight-forward, quantification of $P C A 3$ tissue expression is more challenging. Adding to this difficulty, in contrast to ERG which stains endothelial cells thereby offering a positive internal control, no internal positive control was available for PCA3 RNA in situ hybridization, although successful positive controls (evaluating for the presence of RNA) were run in parallel. Third, PCA3 RNA in situ hybridization was only performed on representative slides containing the majority of the index nodule. Also, although expression of
PCA3 was evaluated in high-grade prostatic intraepithelial neoplasia that happened to be on cancer-containing slides, the present study was not designed to evaluate the $P C A 3$ expression in all high-grade prostatic intraepithelial neoplasia within a prostate. It is possible that unmeasured high-grade prostatic intraepithelial neoplasia burden contributed to urine $P C A 3$, particularly given the majority of high-grade prostatic intraepithelial neoplasia is $P C A 3$ positive. Finally, a small subset $(13 \%)$ of small cancer foci was lost on obtaining deeper sections for PCA3 RNA in situ hybridization. These lost foci may contribute to the lack of correlation between urine and tissue $P C A 3$ expression, to a limited extent.

Consistent with this lack of correlation between urine and tissue $P C A 3$ values, the majority of patients with urine $P C A 3<25$ demonstrated at least one PCA3-positive tumor focus, and half demonstrated an index tumor nodule with strong PCA3 expression (4/4 intensity) by RNA in situ hybridization. Insufficient RNA collection is an unlikely explanation for these discrepant cases, because urine prostate-specific antigen mRNA was measured in all cases, and those with insufficient values were excluded from the study. Urine PCA3 score may reflect associations between tumor burden and overall prostate volume, with larger prostates contributing more PSA mRNA from benign prostatic tissue, thus lowering the PCA3:PSA ratio. This hypothesis will need to be investigated in future studies. False positive tissue PCA3 RNA in situ hybridization is unlikely, as background benign prostate glands served as a successful negative control. A lack of association between in situ hybridization values and transcription median amplification values also appears to be unlikely, as our data show that the values between these different methodologies appear to correlate. On the basis of the preliminary observations from an independent and related study of evaluation of tissue PCA3 expression in cases with very high and very low urine $P C A 3$, we found other cases with similar discrepant results (unpublished data from our group, study in progress). It appears that the process by which PCA3 RNA enters the urine from prostate cancer cells may be more complex than we currently realize. Further work on a large cohort of cases is needed to better understand this relationship.

The present study showed that $88 \%(36 / 41)$ and $76 \%(31 / 41)$ of prostatectomy specimens had at least one tumor focus positive for PCA3 RNA in situ hybridization and ERG immunohistochemistry, respectively. Importantly, 95\% (39/41) of cases had at least one tumor focus positive for either PCA3 or ERG. Both patients with no PCA3-positive or ERGpositive cancer focus had urine PCA3 scores $>25$. Thus, $100 \%$ of patients in this study showed overexpression of ERG or PCA3 in tissue, urine, or both.

The current study has some limitations. This cohort does not reflect the entire spectrum of pathology seen at radical prostatectomy, including 
cases with high Gleason score and high stage. Hence, our results may not necessarily reflect the performance of PCA3 RNA in situ hybridization in high-grade or late-stage cancers. Our cohort also lacks follow-up information precluding the evaluation of associations with outcome, and is relatively small. Hence, our findings regarding the expression of PCA3 by RNA in situ hybridization in prostate cancer need to be validated in a larger series.

\section{Acknowledgments}

This work was supported in part by the US National Institutes of Health Early Detection Research Network (U01 CA111275 and U01 CA113913), NIH SPORE (P50 CA69568), and R01 CA132874. NP and SAT are supported by University of Michigan Prostate SPORE Career Development Awards. AMC is supported by the Howard Hughes Medical Institute, the Doris Duke Foundation, and the Prostate Cancer Foundation and is an American Cancer Research Professor and a Taubman Scholar. We would like to thank Angela Wu, Ritu Bhalla and Rohit Mehra for providing help and advice.

\section{Disclosure/conflict of interest}

AMC serves on the advisory boards of Gen-Probe and Ventana Medical Systems/Roche. SAT has received honoraria from, and consults for Ventana Medical Systems. SAT and AMC are co-inventors on a patent filed by the University of Michigan covering ETS fusions in prostate cancer. The diagnostic field of use has been licensed to Gen-Probe, who has sublicensed some rights to Ventana Medical Systems/Roche. Gen-Probe and Ventana/Roche did not play a role in the design and conduct of this study, in the collection, analysis, or interpretation of the data, or in the preparation, review, or approval of the article. NP does receive research funding from Ventana/Roche but this funding was not used for the development of the assay.

\section{References}

1 Bussemakers MJ, van Bokhoven A, Verhaegh GW, et al. DD3: a new prostate-specific gene, highly overexpressed in prostate cancer. Cancer Res 1999;59: 5975-5979.

2 Schmidt U, Fuessel S, Koch R, et al. Quantitative multi-gene expression profiling of primary prostate cancer. Prostate 2006;66:1521-1534.

3 Popa I, Fradet Y, Beaudry G, et al. Identification of PCA3 (DD3) in prostatic carcinoma by in situ hybridization. Mod Pathol 2007;20:1121-1127.

4 Tomlins SA, Rhodes DR, Perner S, et al. Recurrent fusion of TMPRSS2 and ETS transcription factor genes in prostate cancer. Science 2005;310:644-648.

5 Tomlins SA, Laxman B, Dhanasekaran SM, et al. Distinct classes of chromosomal rearrangements create oncogenic ETS gene fusions in prostate cancer. Nature 2007;448:595-599.

6 Kumar-Sinha C, Tomlins SA, Chinnaiyan AM. Recurrent gene fusions in prostate cancer. Nat Rev Cancer 2008;8:497-511.

7 Mosquera J-M, Perner S, Genega EM, et al. Characterization of TMPRSS2-ERG fusion high-grade prostatic intraepithelial neoplasia and potential clinical implications. Clin Cancer Res 2008;14: 3380-3385.

8 Rubin MA, Maher CA, Chinnaiyan AM. Common gene rearrangements in prostate cancer. J Clin Oncol 2011;29:3659-3668.

9 Tomlins SA, Bjartell A, Chinnaiyan AM, et al. ETS gene fusions in prostate cancer: from discovery to daily clinical practice. Eur Urol 2009;56:275-286.

10 Park K, Tomlins SA, Mudaliar KM, et al. Antibodybased detection of ERG rearrangement-positive prostate cancer. Neoplasia 2010;12:590-598.

11 Tomlins SA, Palanisamy N, Siddiqui J, et al. Antibodybased detection of ERG rearrangements in prostate core biopsies, including diagnostically challenging cases: ERG staining in prostate core biopsies. Arch Pathol Lab Med 2012;136:935-946.

12 Young A, Palanisamy N, Siddiqui J, et al. Correlation of Urine TMPRSS2:ERG and PCA3 to ERG + and Total Prostate Cancer Burden. Am J Clin Pathol 2012;138: 685-696.

13 Tomlins SA, Aubin SMJ, Siddiqui J, et al. Urine TMPRSS2:ERG fusion transcript stratifies prostate cancer risk in men with elevated serum PSA. Sci Transl Med 2011;3:94ra72.

14 Cornu J-N, Cancel-Tassin G, Egrot C, et al. Urine TMPRSS2:ERG fusion transcript integrated with PCA3 score, genotyping, and biological features are correlated to the results of prostatic biopsies in men at risk of prostate cancer. Prostate 2012;73:242-249.

15 Lin DW, Newcomb LF, Brown EC, et al. Urinary TMPRSS2:ERG and PCA3 in an active surveillance cohort: results from a baseline analysis in the Canary Prostate Active Surveillance Study. Clin Cancer Res 2013;19:2442-2450.

16 Stephan C, Jung K, Semjonow A, et al. Comparative assessment of urinary prostate cancer antigen 3 and TMPRSS2:ERG gene fusion with the serum prostate-specific antigen-based prostate health index for detection of prostate cancer. Clin Chem 2013;59: 280-288.

17 Wise AM, Stamey TA, McNeal JE, et al. Morphologic and clinical significance of multifocal prostate cancers in radical prostatectomy specimens. Urology 2002;60: 264-269.

18 Marks LS, Fradet Y, Deras IL, et al. PCA3 molecular urine assay for prostate cancer in men undergoing repeat biopsy. Urology 2007;69:532-535.

19 Braun M, Goltz D, Shaikhibrahim Z, et al. ERG protein expression and genomic rearrangement status in primary and metastatic prostate cancer-a comparative study of two monoclonal antibodies. Prostate Cancer Prostatic Dis 2012;15:165-169.

20 Falzarano SM, Zhou M, Carver P, et al. ERG gene rearrangement status in prostate cancer detected by immunohistochemistry. Virchows Arch 2011;459: 441-447.

$21 \mathrm{He} \mathrm{H,} \mathrm{Magi-Galluzzi} \mathrm{C,} \mathrm{Li} \mathrm{J,} \mathrm{et} \mathrm{al.} \mathrm{The} \mathrm{diagnostic} \mathrm{utility}$ of novel immunohistochemical marker ERG in the workup of prostate biopsies with 'atypical glands 
suspicious for cancer'. Am J Surg Pathol 2011;35: 608-614.

22 Jenkins RB, Qian J, Lee HK, et al. A molecular cytogenetic analysis of 7q31 in prostate cancer. Cancer Res 1998;58:759-766.

23 Qian J, Bostwick DG, Takahashi S, et al. Chromosomal anomalies in prostatic intraepithelial neoplasia and carcinoma detected by fluorescence in situ hybridization. Cancer Res 1995;55:5408-5414.

24 Vukovic B, Park PC, Al-Maghrabi J, et al. Evidence of multifocality of telomere erosion in high-grade prostatic intraepithelial neoplasia (HPIN) and concurrent carcinoma. Oncogene 2003;22:1978-1987.

25 Kunju LP, Chinnaiyan AM, Shah RB. Comparison of monoclonal antibody (P504S) and polyclonal antibody to alpha methylacyl-CoA racemase (AMACR) in the work-up of prostate cancer. Histopathology 2005;47: 587-596.

26 Auprich M, Bjartell A, FK-H Chun, et al. Contemporary role of prostate cancer antigen 3 in the management of prostate cancer. Eur Urol 2011;60:1045-1054.

27 Groskopf J, Aubin SMJ, Deras IL, et al. APTIMA PCA3 molecular urine test: development of a method to aid in the diagnosis of prostate cancer. Clin Chem 2006;52: 1089-1095.

28 Ploussard G, Durand X, Xylinas E, et al. Prostate cancer antigen 3 score accurately predicts $t$ umour volume and might help in selecting prostate cancer patients for active surveillance. Eur Urol 2011;59: 422-429. 